\title{
An Intelligent Learning Agent using Learner's Information in Mobile Environment
}

\author{
Jin-Il Kim \\ Dept. of Mathematics Education, Hannam University, 133 Ojeong-dong, \\ Daedeok-gu, Daejon 306-791, Korea \\ jikimi@hnu.kr
}

\begin{abstract}
Recently, Since people always carry mobile devices and can learn whenever and wherever by using not only phone, text, SNS, and TV, but also using learning device, recorder, e-book, e-dictionary, and internet surfing due to rapid distribution of smartphone, the interest in intelligent learning agent related to situated learning is increasing more. The method of practicing the situated learning theories developed so fan provides learning contents to learners by analyzing their environment information along with their basic information. However, if learning contents are recommended by considering only basic and partial information of a learner, in many cases, they do not match with the demand from the learner, so it could become factors that decrease lamer's learning desire and obstruct inducing learning motivation. Therefore, this studx propose@intelligent learning agent that automatically recommends learning contents that learners want in timely manner in their real life by analyzing not only learners'docation information, but also their available time for learning and Internet activity pattern
\end{abstract}

Keywords: Situated Learning, Intelligen Learning Agent, Internet activity pattern

\section{Introduction}

Smartphones and tablet PC are changing people's life pattern as they become popular due to rapid development of information and communication technology (ICT). Just as much IT devices have very close relationship with our lives. The new technology that could be only seen in movies can now be used easily by anyone even in education, so changes are occurring in learning by using mobile devices.

Learning by using mobile devices is knowledge acquired through interaction in the context of real life, so it is practical and is to transfer to problem solutions in reality. Since learners could accumulate knowledge along with practical experiences, it could be said that it follows the practicing methods of situated learning theory [1].

Although the learning by using mobile device could provide an environment where knoyledge is transferred real-time through wireless internet, for learners to learn appropriate for their situation, they need to directly download appropriate learning contents from web servers or search for necessary contents by themselves among the contents stored in mobile device. In order to solve such inconvenience, studies on intelligent learning agent related to situated learning are going on actively [1-3].

However, the intelligent learning agents developed so far mostly provided learning contents to learners by analyzing environment information along with learners' basic information. Thus, if learning contents are recommended by considering only basic and partial information of learners, many times they do not accord with learners' demand, so it 
could be a factor that decrease learners' desire for studying and inhibit inducing motivation for studying. Therefore, this study provides an intelligent learning agent that automatically recommends studying contents that learners want in their real life by considering not only learners' environment information, but also their learning ability information and internet life pattern.

\section{Related Study}

\subsection{Situated Earning}

Situated learning in mobile environment could be said as one of the new learning methods that are receiving the most attention nowadays. As mobile technology brought innovative changes in social and cultural fields just like when internet was firrst introduced in education field, the studies for using mobile technology in situated learning are going on actively.

According to situated learning, the learning that occurs in the context of real life connects the learning contents with learners' personal experience and dêmand, so not only does it give motivation for learners to learn bur also enables them to understand knowledge more easily. Also, the knowledge acquired through context is more practical and easy to transfer over to problem solving in real life than the knowledge acquired through direct teaching $[4,5]$.

According to situated learning, people could gain knowledge with vicarious experiences for situation and context of rear ife in a yirtual space similar to reality [6]. The Cognition and Technology Group at Vanderbilt University developed Jasper series that is a concrete program that applied situated learning principle and it to students, and as a result, it proved that the students problem solving ability improved [7,8].

Also, the study of applying situated learning to the virtual environment of online progressed actively, owing to the advancement of information technology [9]. There are studies with a purpose of inducing motivation for studying by considering game's fun and interest factors and MMORPQ playing game system with the characteristic of virtual social space which is more than a simple game dimension [10]. However, in a strict sense, itis difficult to say that anchored instruction that consists of interactive video format or learning that occurs in a virtual world of online are teaching methods that completely accord with situated learning. It is because although such teaching methods provide contex similar to reality, it delivers learning contents unilaterally, and from learners' perspective, they learn by indirect experience through observation, not direct experience

Recently, the study on the supportive system that enables learning in the actual site, outside classrooms, by using mobile device is going on actively [11-15]. When several study cases related to it are examined, there are mobile learning contents design that supports situated learning by using cell phone device with wireless internet [16], study tha realized learning contents in mobile device in a isolated form [3], and mobile learning system that can be used after downloading necessary contents to PDA from web server [17].

\subsection{Intelligent Agent}

Intelligent agent is a system that attempts to achieve purpose autonomously to some degree in complex environment that changes, and it has been studied in artificial intelligent field under names as autonomous agent, software agent, and interface agent. 
The method of manually designing intelligent agent requires designer to have sufficient knowledge about application domain, and it also has a problem of system function being fastened early on. In order to overcome such problem, the method of designing intelligent agent that uses machine learning that develops algorithm automatically is attempted $[18,19]$.

Machine learning is a technology that generates all kinds of program by extracting rule or pattern from large data set, and it uses neural network, decision tree, genetic algorithm, SVM (Support Vector Machine), and Bayesian Network [20]. The method of intelligent agent design by using machine learning consists agent based on the information collected from environment, so it can be applied in diverse areas, and although environment or propensity of user change, it sustains the quality of work by changing the agent adaptively.

When several studies that applied machine learning technique to intelligent agent, they modeled the propensity of user with decision tree and applied to fire agent that manages meeting schedule [18], and it learned the propensity of user by optimizing fuzzy model that designs meeting schedule by using genetid afgorithm 121]. Schiaffino and Amandi learned users' propensity or habit and provided proper alarm service by using reinforcement learning [22].

Intelligent agent that performs information search introduced machine learning to understand user intention more accurately, it automatically searched the expert of problems that users intended to solve by using collaborative filtering [23], and it suggested the agent that searches customized information by analyzing users' search tendency with genetic algorithm [24] Horvitz et al applied interactive agent, which deduces user intention through natural language by using Bayesian Network and provides relevant information, to MS-Office [25], and they modeled expert knowledge with neural network in information customization system based on multiple agents [26].

Therefore, this study develops intelligent learning agent based on Bayesian Network, which is collaborative filteng method based on model, so that it would be appropriate for mobile environmen that requires rear-time data handling.

\section{The Proposed Lutelligent Learning Agent}

The structure of intelligent learning agent proposed by this study consists of context awareness module, learning recommendation module, and system mapping module like Figure 1.

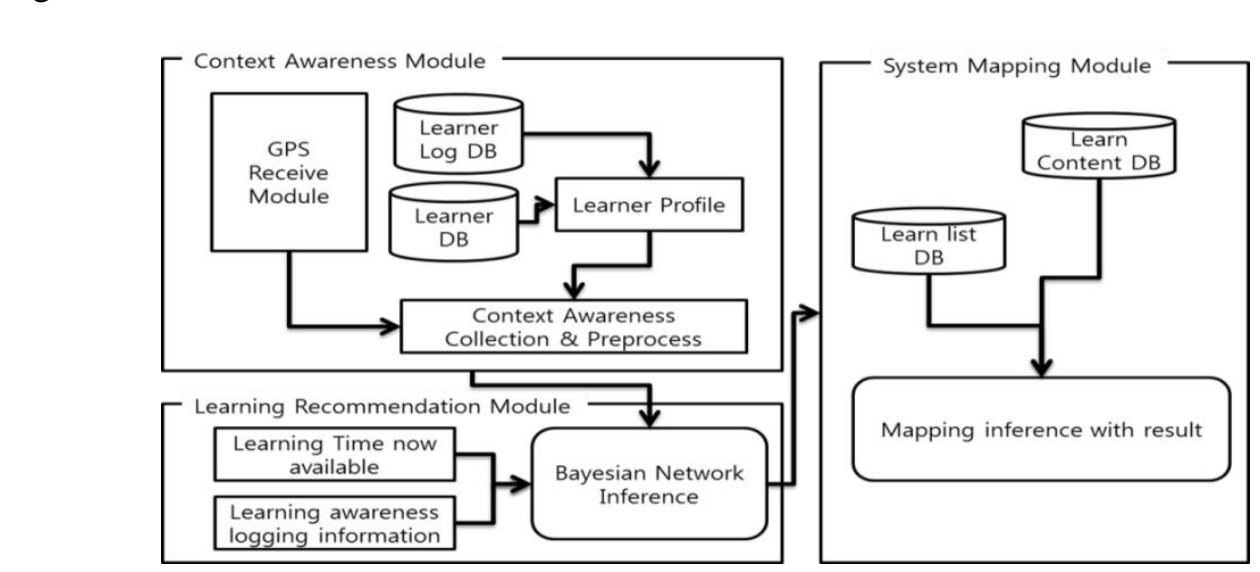

Figure 1. The Proposed System 


\subsection{Context Awareness Module}

Context awareness module combines learners' location information by using GPS signal and learning ability information and provides learning contents appropriate to learners' current situation. First, it collects individual learning ability information stored in learners' mobile device and context information by using location information GPS. It changes the collected information appropriate to each node's name and state for the parameter learning of Bayesian Network and performs. Each node conducts parameter learning based on preprocessed context information and obtains conditional probability table. When the location of learners moves, it selects top data group with top probability accordingly by using Bayesian Network model that is parameter-learned. It constructs real learning information that accords with the selected group through contents database search.

\subsection{Learning Recommendation Module}

Learning recommendation module is the core of the proposed system, and it intelligently recommends learning contents provided to learners based on the result of learning time now available module and log information collection module.

\section{A. Learning Time Now Available Module}

Basically, learning contents should be recommended in the free time of learners when they can study. Also, it should guarantee so that recommended fearning contents could be completed within the given time to efficiently manage such learning time. In other words, ideal learning scheduler schedules so that learners would complete appropriate learning contents that are recommended in their free time within the expected learning time. If learning is completed in the freetine, there is no need to store the intermediate information of the recommended learning contents or to reschedule. In order to match learning contents with learners' available time for learning it needs to consider not only the method to predict learners' available time for learning, bat also the method to expect appropriate contents during that time period.

In order to predict learners' avaitable time for learning, first use intuition. Learners have the tendency to stady at the same time everyday and the tendency to have predictable idle time during the day. In other words, learning is done during commute, lunch time, weekends, and holidays. By using such observation, it could find the available time for learning by recent time unit, day unit, daily unit, and weekly unit. It has important correlation with historical usage pattern

In order to schedure learning, it is very important to predict learners' available time for learning for the next $n$ minute. Here, $n$ shows the available time for learning. This technique emphasizes learners' average available time for learning.

The method that predicts learner's available time for learning assumes that the available time for the next learning ( $\mathrm{n}$ minute) would precisely accord with the previous learning time (n_minute). Here, there are three methods based on the RecentT_ST, Day_ST, and Week_ST. The RecentT_ST method predicts that the predicted time available for learning recently ( $\mathrm{n}$ minute) accords precisely with the last learning time (n minute). For example, if the average learning time is four minutes during the previous twenty minutes $(n=20)$, then the RecentT_ST method predicts that the average learning time would be four minutes for the next twenty minutes. The Day_ST and Week_ST methods are similar to RecentT_ST method except that they use different standards to predict learning available time. The Day_ST method predicts that the next time available for learning ( $\mathrm{n}$ minute) would be exactly the same as the $\mathrm{n}$ minutes from the previous day. For example, in case of studying language, 
people tend to learn at the same time everyday like during the commute or lunch time. The Week_ST method predicts that the next time available for learning would accord with the $\mathrm{n}$ minute of the same time last week.

As the result of the experiment (Figure 2), the best ways to predict the learner's available time for learning are generated at the previous $\mathrm{n}$ minute when it is less than ten minutes $(\mathrm{n} \leq 10)$ (the RecentT_ST method), and at the time in the previous day when it is more than ten minutes $(n>10)$ (the Day_ST method). The prediction method with the lowest accuracy rate is the Week_ST method. It can be learned that for the scheduling algorithm that predicts available time for learner, if the value of $n$ is small, then it can be made excellent by using recent information.

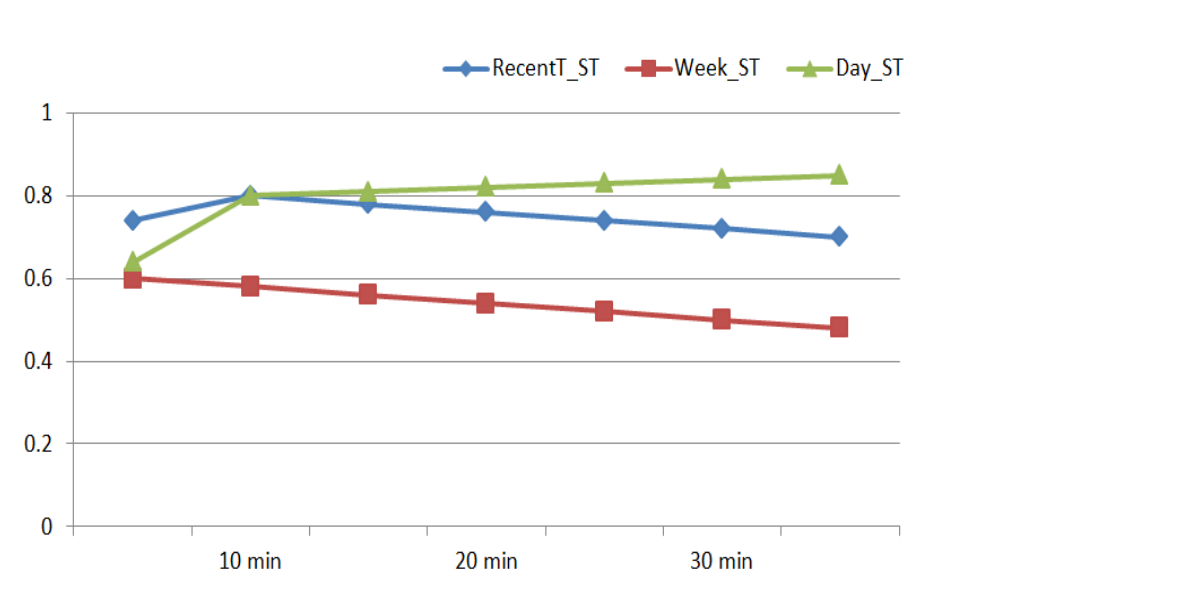

Figure 2. Learhing Time Prediction Methods

B. Learning Awareness Logging Information Module

\begin{tabular}{c|l}
\hline Lerm & \multicolumn{1}{c}{ definition } \\
\hline Extraction set & set of the cases regarding the extracted keyword \\
\hline Extraction & Keyword that becomes the main at a certain time period \\
\hline Recent keyword & keyword that becomes the main of the day \\
\hline Daily keyword & keyword that becomes the main of the day \\
\hline Weekly keyword & keyword that becomes the main of the week \\
\hline Case & Learner's use pattern \\
\hline Main case & the case that had crucial influence on deciding the keyword \\
\hline
\end{tabular}

Collect the leaner's basic information during the process of logging in and all the information regarding learner's use pattern that generates as the learner uses the mobile. The result is stored in the $\log \mathrm{DB}$ and deduce the learner's main interest from the current perspective by using this result. 


\subsection{System Mapping Module}

It is difficult to know which learning contents to choose directly only with the probability values deduced by the deduction results of the Bayesian Network. Therefore, this study intelligently recommends the learning contents provided to learners by considering the results of the context awareness module, learning time now available module, and learning awareness logging information module.

\section{System Implementation and Evaluation}

\subsection{Overview of the Experiment}

The environment for developing mobile learning support system is like Table 1. Since this study develops each module by using Visual $\mathrm{C}++$, it leads the design with an object-oriented model, which can confirm all the defined process and express the system' characteristics well, as its basic.

Mobile learning support system materialized in this way was evaluated with the experiment group of twenty learners who gathered for learning according to the learners' situation information. And it confirmed whether the learning contents, which were automatically recommended to the learners when they had available time for learning, were fit.
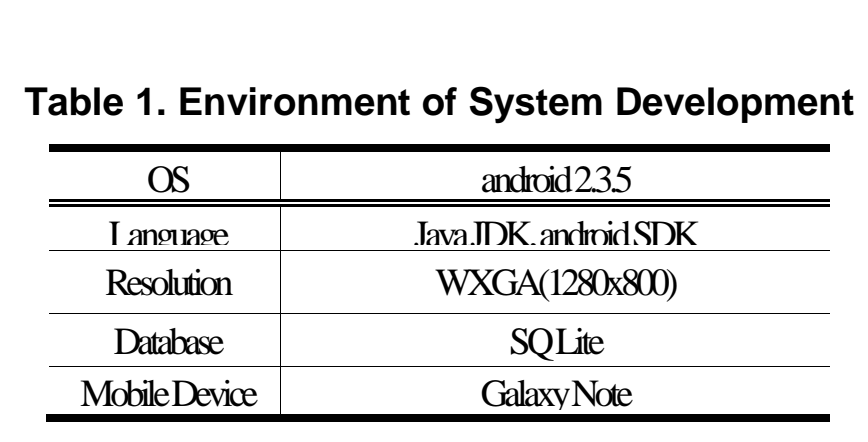

In the collaborated filtering based on learner, the goodness of fit regarding learner's learning contents (s extracted like Table 2 by considering the learner's situation information (location, information on learner's learning capacity, and etc.), and converts it into the scores of goodness of fit regarding learner's learning contents by using Pearson's correlation coefficient like Formula) $[9]$.

$$
L P_{i}=\frac{\sum_{k}\left(L_{i k}-\bar{L}_{\imath}\right)}{\sqrt{\sum_{k}\left(L_{i k}-\bar{L}_{\imath}\right)^{2}}}
$$

Here $L_{i k}$ indicates the goodness of fit of Learner $i$ to $k$, which is the types of learning contents, and $\bar{L}_{l}$ indicates the average of the goodness of fit of Learneri. $L P_{i}$ has a value closer to 1 When the goodness of fit of learner is similar; when the goodness of fit is conflicting, it has a value closer to -1 . Table 2 is an example of a table of goodness of fit in random situation. 
Table 2. Learning Contents Type Preference

\begin{tabular}{c|c|c|c|c|c}
\hline & TO4_A & TE6_B & TE8_C & TE9_D & Avg. \\
\hline Learner 1 & 0.6 & 0.8 & 0.3 & 0.6 & 0.58 \\
\hline Learner 2 & 0.8 & 0.7 & 0.1 & 0.8 & 0.60 \\
\hline Learner 3 & 0.7 & 0.5 & 0.4 & 0.5 & 0.53 \\
\hline$\ldots$ & $\ldots$ & $\ldots$ & $\ldots$ & $\ldots$ & $\ldots$ \\
\hline Learner 18 & 1 & 0.7 & 0.9 & 0.8 & 0.85 \\
\hline Learner 19 & 0.8 & 0.9 & 0.7 & 0.7 & 0.78 \\
\hline Learner 20 & 1 & 0.4 & 0.6 & 0.5 & 0.63 \\
\hline
\end{tabular}

At the phase of calculating preference, get the final recommended learning contents by using the available time for learning and learning awareness logging informat on based on the preference according to learning capacity.

$$
\begin{gathered}
F P_{\text {time }}=\left(L P_{i} \times T_{e 1}\right)+\left(L P_{i} \times T_{e 2}\right)+\cdots+\left(L P_{i} \times T_{e n}\right) \\
F P_{\text {time-log }}=\left(F P_{\text {time }} \times \log _{e 1}\right)+\left(F P_{\text {time }} \times \log _{e 2}\right)+\cdots+\left(E P_{\text {time }} \times \log _{\text {en }}\right)
\end{gathered}
$$

Here, $T_{e 1}, T_{e 2}, \ldots, T_{e n}$ indicates the weighr regarding the avallable time for learning that could have influence on learning, and $\log _{e 1}, \log _{e 2}, \ldots, \log _{e n}$ indicates the weight according to the learning awareness logging information.

By using the goodness of fit of learning contents, the available time for learning, and learning awareness logging information, recommend the contents that learners should ultimately learn like the formula shows.

$$
Q_{\mathrm{LR}}=L P_{i} \otimes E P_{\text {time }} \otimes F P_{\text {time }-l o g}
$$

Generate the recommendation ist in order of high to low LR(Learning_Recommendation) values and provide it to users.

\subsection{Operation Test}

As the result of the experiment, the case of recommending the learning contents by only using the basic sicuation information (1), the case of providing after adding the predictable available time for learning information to the basic situation information (1)+(2), and the case of providing after adding the learning awareness logging information to the basic situation information and available time for learning (1)+(2)+(3) are compared, as the Figure shows. The precision of the information of learning recommendation increased in average of $5 \%$ and $13 \%$ respectively more than that of providing the basic situation information. In the overall average, the precision for (1)+(2) showed the performance of $74 \%$ and $78 \%$ for (1) + (2) + (3). 


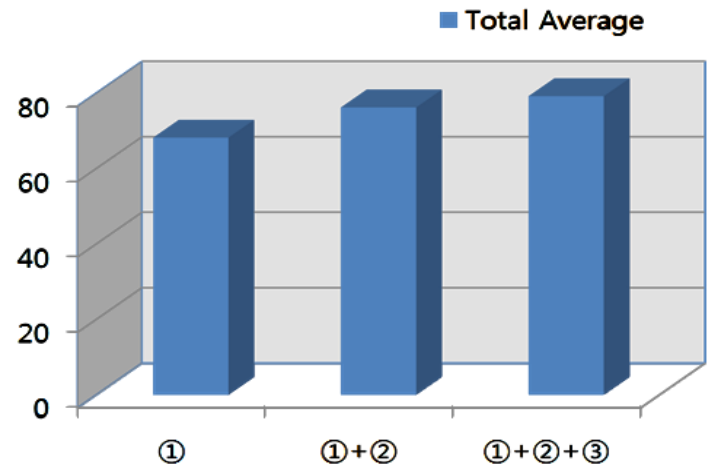

Figure 3. Experiment Results of Recommendation Service

The experiment result like this indicates that when 100 learning recommendations are given, 78 of them would be precise to the learners of the experiment.

Also, this study targeted the limited number of learning contents an a small number of learners for experiment. But if more information of learning contents are collected, then more precise recommendation service would be possible since various learning contents that are appropriate to learner's demands according to situations could betrecommended.

\section{Conclusion}

This study suggests a filtering method for recommendation service system that considered learner's situation. For this, 10 recommends the contents that learners should ultimately learn by using the goodness of fit of learners' learning contents, available time for learning, and learning awareness logging information.

As the result of experimenting by using the filtering method proposed by this study, precision showed $78 \%$ of performance the case of providing after adding the learning awareness logging information to the basic situation information and available time for learning. It increased $13 \%$ more than the method of only providing the existing situation information and $5 \%$ more than the method of adding available time for learning to the basic situation information.

The recommendation serve system that uses the filtering method, which this study suggests, is expected to be useful to not only the learning contents area, but also to the system that reflects the user's tendency for deciding what to recommend. Also, in order to increase precision of recommendation, multilateral analysis on the system is required, and more collection of data is necessary for making more elaborate model.

\section{Acknowledgments}

This paper has been supported by 2015 Hannam University Research Fund.

\section{References}

[1] J.-I Kim, I.-W. Park and H.-H. Lee, “An Intelligent Context-Aware Learning System based on Mobile Augmented Reality”, UCMA2011.Part II, CCIS 151, pp. 255-264, Springer, Deajeon, KOREA, (2011).

[2] J.-I. Kim, Y.-H. Lee and H.-H. Lee, "Development of a Mobile Language Learning Assistant System Based on Smartphone”, FGCN2010.Part II, CCIS 120, Springer, Heidelberg, (2010), pp. 3211-329.

[3] J.-H. Park, "Design and Implementation of Mobile Learning Contents for Situated Learning", Graduate School of Education, Korea National University of Education, Chung-Buk, Korea, (2005). 
[4] J. D. Bransford and N. J. Vye, "A perspective on cognitive research and its implications for instruction", L. B. Resnick\& L. E. Klopfer(Eds.), Toward the thinking curriculum: Current cognitive research, Alexandria VA: Association for Supervision and Curriculum Development, (1989), pp. 173-205.

[5] Cognitive and Technology Group at Vanderbilt. Anchored instruction and situated cognition revisited. Educational Technology, vol. 33, no. 3, (1993), pp. 52-70.

[6] R. Spiro and P. Feltovich, "Cognitive flexibility, constructivism, and hypertext", Educational Technology, vol. 31, no. 5, (1991), pp. 24-33.

[7] Cognitive and Technology Group at Vanderbilt. Anchored instruction and its relationship to situated cognition. Educational Researcher, vol. 19, no. 6, (1990), pp. 2-10.

[8] Cognitive and Technology Group at Vanderbilt. The Jasper experiment: An exploration of issues in learning and instructional design. Educational Technology Research and development, vol. 40, no. 1, (1992), pp. 6580.

[9] Y.-W. Jang, "Design and implementation of the just-in-time learning system applying situated learning theory", Graduate School of Education, Korea National University of Education, Chung-Buk, Korea, (2006).

[10] S.-I. Park, "Development of a situated learning support system based on MMORPG", Graduate School of Education, Korea National University of Education, Chung-Buk, Korea, (2005).

[11] J.-H. Kim, "Design and Implementation of a PDA-based Mobile Learning Contents -Centering on Wordprocessor Learning-.Graduate School of Education”, Catholic University of Daegu, Daegu, Korea, (2004).

[12] K.-M. Yang, C.-M. Kim and S.-B. Kim, "The Desing and Implementation of an Engish Situated Learning System based on RFID”, Journal of The Koran Association of Computer Education, vol. 9, no. 6, (2006), pp. 65-78.

[13] J.-S. Sung, “U-Learning Model Design Based on Ubiquitous Environment”, JAST, vol. 13, (2009), pp. 7788.

[14] J. Lee and Y.-J. Lee, "Development and Application of E-Learning Content for Advertising Education", IJAST, vol. 47, (2012), pp. 1-12.

[15] S. Jee and H.-C. Kim, "The Practical Application of Computer-Assisted Language Learning in English as a Foreign Language", IJSEIA, vol. 6, no. 4, (2012), pp. 165-170.

[16] J.-H. Jeong, "Design and development of mobile learning contents for field activities", Graduate School of Education, Korea National University of Education, Chung-Buk, Korea, (2004).

[17] J.-C. Park, “A Design and Implementalon of Experiment-Practice Study Contents using a PDA", Graduate School of Education Silla University, Korea, (2003).

[18] T. Mitchel, "Experience with a learning personal assistant", Communication of the ACM, vol. 37, no. 7, (1994), pp. 80-91.

[19] T. E. Rothenfluh, "Reusable ontologies, knowledge-acquisition tools, and performance systems: PROTE'GE'-II solution to Sisyphus-2", International Journal of Human-Computer Studies, vol. 44, no. 3-4, (1996), pp. 303-332.

[20] J.-H. Hong and S.-B. Cho, "Machine Learning and Intelligent Agents", Communications Of The Korea Information Science Society, ol 25, no. 3, (2007), pp. 64-69.

[21] C. Lee and C. Pan, "An intelligent fuzzy agent for meeting scheduling decision support system", Fuzzy Sets and Systems, vol. 142, no 3, (2004), pp. 467-488.

[22] S. Schiaffino and A. Amand, "Polite personal agents", IEEE Intelligent Systems, vol. 21, no. 1, (2006), pp. $12-19$.

[23] H. Kautz, "Referral web: Combining social networks and collaborative filtering", Communication of the ACM, vol. 40, no 3, (1997), pp. 63-65.

[24] C. Hsinchun, "An intelligent personal spider(agent) for dynamic internet/intranet searching", Decision Support-Systems, vol. 23, no. 1, (1998), pp. 41-58.

[25] E. Horvitz, "The Lumiereproject: Bayesian user modeling for inferring the goals and needs of software users", Proc. 14th Conf. on Uncertainty in Artificial Intelligence, (1998).

[26] M. Hamdi, "MASACAD: A multi agent-based approach to information customization for the purpose of ademic advising of students", Applied Soft Computing, vol. 7, no. 3, (2007), pp. 746-771. 


\section{Author}

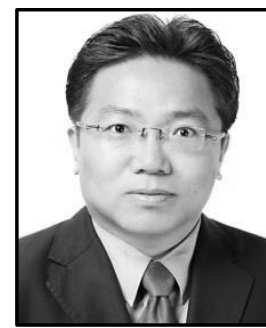

Jin-Il Kim, received the $\mathrm{BS}$, the $\mathrm{MA}$ and the $\mathrm{PhD}$ degreesin computer science from Hannam University, Korea, in 1991, 1993 and 2000, respectively.AlsoHe received the $\mathrm{MA}$ and the $\mathrm{PhD}$ degreesin Education from Korea University, Korea, in 2003 and 2009,respectively.His research interestsinclude Parallel Processing \&Network, Situated Learning and u-Learning.

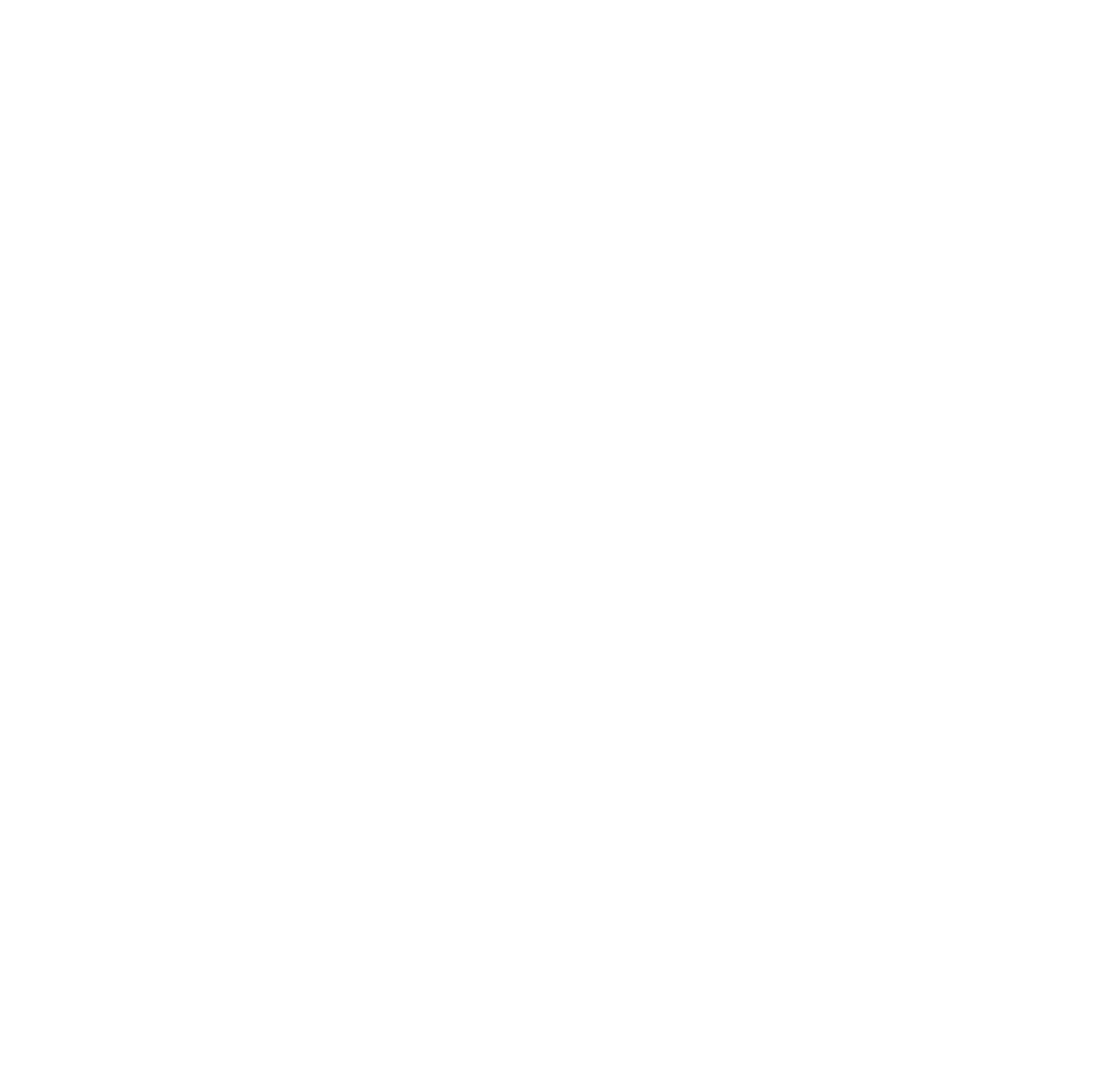

